\title{
Comparative efficacy of respiratory personal protective equipment against viral respiratory infectious diseases in healthcare workers: A network meta-analysis
}

\author{
Xinbo Yin ${ }^{1}$, Xiaokai Wang $^{2}, \mathrm{Su} \mathrm{Xu}^{3}$, and Caiyun $\mathrm{He}^{1}$ \\ ${ }^{1}$ Hunan Normal University \\ ${ }^{2}$ Xiangya Hospital Central South University \\ ${ }^{3}$ The University of Melbourne Melbourne Dental School
}

July 31, 2020

\begin{abstract}
Background: With the epidemic of COVID-19, the health care workers (HCWs) require proper respiratory personal protective equipment (rPPE) against viral respiratory infectious diseases. However, there is little published evidence comparing their efficacy. Therefore, we conducted a network meta-analysis (NMA) to compare the efficacy of rPPEs and the wearing manner preventing from the viral respiratory infectious diseases (VRID). Methods: This NMA protocol was registered in PROSPERO (CRD42020179489). PubMed, the Cochrane Library, Web of Science, and EMBASE were searched by computer to collect cluster randomized control trials of comparing the effectiveness of rPPEs and wearing manner in preventing HCWs from VRID. The primary outcome was the incidence of laboratory-confirmed viral respiratory infection. The secondary outcome was the incidence of CRI. The Bayesian NMA was conducted to use the control group as the comparison group. Incidence of the VRID and CRI were reported as network odds ratio (network OR) and 95\% credibility interval (CrI). Results: Six studies encompassed 11,828 HCWs and seven interventions were included. In terms of the incidence of laboratory-confirmed viral respiratory infection, the continuous wearing of N95 respirator (network OR, 0.48; 95\% CrI 0.27 to 0.86; SUCRA score, 85.4) showed more effective than the control group. In terms of the incidence of CRI, there are no interventions that showed more effective than the control group. Conclusion: There are significant differences in preventive efficacy among current rPPEs. Our result suggests that continuous wearing of N95 respirator can serve as the best preventive rPPE for HCWs from the VRID.
\end{abstract}

Title

Comparative efficacy of respiratory personal protective equipment against viral respiratory

infectious diseases in healthcare workers: A network meta-analysis

Running title

Evaluation of the efficacy of respiratory personal protective equipment by the network meta-analysis

Authors

Xinbo Yin $^{1.2}$ B.S.

Xiaokai Wang ${ }^{2}$ M.D.

$\mathrm{Su} \mathrm{X} \mathrm{X}^{3}$ D.D.S.

Caiyun $\mathrm{He}^{1 *}$ M.S. ${ }^{*}$ Corresponding Author. E-mail: hecaiyunhnu@126.com

Author Contributions 
C.H.: Conceptualization (lead); writing - review and editing (equal).; X.B: Writing - Methodology (lead); Software (lead); formal analysis (lead); writing - review and editing (equal). X.W.: Writing - original draft (lead); writing - review and editing (equal). S.X.: writing - review and editing (equal).

Author affiliations:

1. Department of nursing, medical college, Hunan Normal University, Changsha, Hunan. China.

2. Department of Emergency Medicine, Xiangya Hospital, Central South University. Changsha,

Hunan. China.

3. Melbourne Dental School, University of Melbourne, Parkville, Victoria, Australia

Sources of financial support

$\mathrm{N} / \mathrm{A}$

Conflict of interest

The authors have no conflicts of interest to declare.

Comparative efficacy of respiratory personal protective equipment against viral respiratory infectious diseases in healthcare workers: A network meta-analysis

\begin{abstract}
Background: With the epidemic of COVID-19, the health care workers (HCWs) require proper respiratory personal protective equipment (rPPE) against viral respiratory infectious diseases. However, there is little published evidence comparing their efficacy. Therefore, we conducted a network meta-analysis (NMA) to compare the efficacy of rPPEs and the wearing manner preventing from the viral respiratory infectious diseases (VRID).
\end{abstract}

Methods : This NMA protocol was registered in PROSPERO (CRD42020179489). PubMed, the Cochrane Library, Web of Science, and EMBASE were searched by computer to collect cluster randomized control trials of comparing the effectiveness of rPPEs and wearing manner in preventing HCWs from VRID. The primary outcome was the incidence of laboratory-confirmed viral respiratory infection. The secondary outcome was the incidence of CRI. The Bayesian NMA was conducted to use the control group as the comparison group. Incidence of the VRID and CRI were reported as network odds ratio (network OR) and $95 \%$ credibility interval (CrI).

Results : Six studies encompassed 11,828 HCWs and seven interventions were included. In terms of the incidence of laboratory-confirmed viral respiratory infection, the continuous wearing of N95 respirator (network OR, 0.48; 95\% CrI 0.27 to 0.86; SUCRA score, 85.4) showed more effective than the control group. In terms of the incidence of CRI, there are no interventions that showed more effective than the control group.

Conclusion : There are significant differences in preventive efficacy among current rPPEs. Our result suggests that continuous wearing of N95 respirator can serve as the best preventive rPPE for HCWs from the VRID.

\title{
Keywords
}

Viral respiratory infectious diseases; Surgical mask; N95 respirator; Network meta-analysis; Health care workers. 


\section{Introduction}

Respiratory personal protective equipment is critical to reducing the risk of spreading respiratory pathogens in the current COVID-19 epidemic. ${ }^{1}$ There are three main types of rPPE currently used in HCWs: cloth mask, surgical mask, and N95 respirator. Cloth mask, although it has been replaced by the disposable surgical mask, is still used repeatedly in the area wherein the shortage of rPPE. The surgical mask has traditionally been used by HCWs to avoid hand-to-face contact and prevent respiratory droplet transmission, although it may not be reliable for preventing aerosol transmission ${ }^{2}$. The N95 respirator is designed to prevent HCWs from inhaling small airborne particles when treating suspect VRID patients ${ }^{3}$. In the epidemic of COVID-19, N95 respirator is strongly recommended to use in HCWs from occupationally acquired infections through droplet or airborne spread. ${ }^{1}$

Although N95 respirator has been thought to be superior to other masks (e.g. surgical mask) in preventing the COVID-19 as well as the other common VRID (e.g. influenza, respiratory syncytial virus, metapneumovirus, parainfluenza virus, rhinovirus-enterovirus, coronavirus, etc.), the existing evidence is still controversial. ${ }^{4,5}$ This may be due to the diagnosis of VRID replies on laboratory tests based on nucleic acid or antibody, however, false-negative results do exist and may undermine the sensitivity of the test. To minimize the risk of missing specific targets and increase the sensitivity, clinical respiratory illness (CRI) is an important supplemental method to diagnose VRID. We include the data from laboratory tests as well as clinical presentations (e.g. fever, respiratory rate $>25 / \mathrm{min}$, etc.) and symptoms (e.g. chills, cough, diarrhea, dyspnea, fatigue, headache, etc. $)^{6}$ in this NMA to better evaluate and compare the protective effects of rPPEs.

It is noteworthy that asymptomatic infections existed in many VRID. So, the wearing manner is also important for HCWs who were facing the suspectable patient. ${ }^{7-9}$ There are two types of wearing manners including continuous wearing and targeted wearing. Continuous wearing refers to wearing of the rPPE during the whole working shift, whereas targeted wearing refers to wearing only on performing high-risk procedures (e.g. endotracheal intubation) or in high-risk situations (e.g. entering an isolation room or barrier nursing of a patient). ${ }^{10}$ The previous study also found out that different wearing manners affected the protective efficacy of rPPE. ${ }^{10}$ Recently, Bartoszko et al. provide a negative result in comparing surgical masks and N95 respirators in COVID-19 epidemics. ${ }^{5}$ However, the manner of wearing might take consideration in preventive efficacy in rPPE. Hence, we conducted this network meta-analysis to examine which type of mask and manner of wearing is the best suitable rPPE for preventing the VRID.

\section{Methods}

\section{Data Sources and Search Strategy}

This network meta-analysis (NMA) protocol was registered in PROSPERO (CRD42020179489). This NMA was conducted following guidelines in the preferred reporting items for systematic reviews and meta-analyses (PRISMA) report, the extension of network meta-analyses ${ }^{11}$.PubMed, the Cochrane Library, Web of Science, and EMBASE were searched by computer to collect cluster randomized control trials of comparing the effectiveness of rPPEs and wearing manner in preventing HCWs from VRID. The retrieval time limit was from Jan 1, 1970, to Dec 31, 2019. Simultaneously, the research and related systematic evaluation references that have been included in the manual retrieval are conducted to supplement and obtain relevant literature. Through PubMed, the search strategy is determined, and the search is carried out with a combination of subject words and free words. English search terms include the random controlled trial (RCT), mask, face mask, respirator trace effects, respirator masks, N95 respirator masks, and virus, etc.

\section{Study Selection and Eligibility Criteria}

Inclusion criteria were cluster RCTs comparing the effectiveness of rPPEs and wearing manner in preventing HCWs from VRID. The outcome includes the incidence of laboratory-confirmed viral respiratory infection and the incidence of clinical respiratory illness (Table 1). Exclusion criteria were non-RCT experiments; 
incomplete or repeated publication of relevant data; non-human studies; and reviews, study protocols, comments, case reports, and letters.

\section{Data extraction and quality assessment}

Two authors (XB. Y. and XK. W.) independently extracted relevant data parameters. In case of disagreement, the arbitration shall be conducted by the corresponding author. The following data extraction parameters were extracted: name of the primary author, country of study, number of HCWs, number of participants per arm, HCW age (mean or median and standard deviation [SD] or range, if available), the gender of HCWs, quality information included in the study, the efficiency of the incidence of laboratory-confirmed viral respiratory infection in $\mathrm{rPPE}$ arm and control arm, the efficiency of the incidence of clinical respiratory illness in rPPE arm and control arm.

The study quality was assessed by two authors (XB. Y. and XK. W.) according to Cochrane Collaboration's tool. It includes six aspects: sequence generation, allocation consideration, blinding, incomplete outcome data, no selective outcome reporting, other sources. RevMan software ( $\mathrm{v}$ 5.3) was only used for the risk of bias summary. In case of disagreement, the arbitration will be conducted by the corresponding author.

\section{Outcomes}

The prespecified primary outcome was the incidence of laboratory-confirmed viral respiratory infection reported as an odds ratio (OR) value and $95 \%$ credibility interval $(\mathrm{CrI})$. The OR value was calculated by taking the odds of laboratory-confirmed viral respiratory infection in a specific rPPE group and dividing this value by odds of the control. The prespecified secondary outcome was the incidence of clinical respiratory illness reported as an OR with the associated $95 \%$ CrI. The OR was calculated by taking the odds of CRI in a specific rPPE group and dividing this value by odds of the control. The protective effect of rPPE was defined as an OR (including the associated 95\% CrI) falling under unity (1.0).

\section{Statistical Analysis}

The Stata15 SE was used for network diagrams. The ADDIS software (version1.16.8) was used for node-split analysis and consistency test of direct and indirect comparison. If P-value was $>0.05$, the random model consistency type was used for analysis, otherwise, use the random model inconsistency type, If can't use node-split analysis, provide both consistency type and inconsistency type data. ${ }^{12}$ Then using PSRF analysis to determine the model convergence, when PSRF value was 1 indicates approximate convergence has been reached, ${ }^{13}$ finish the analysis and selected Network OR value and $95 \%$ CrI as the effect magnitude, output ranks, and the SUCRA (surface under the cumulative ranking curve analysis) value.

To determine the heterogeneity and result stability, The R software (version 3.6.1) was used for heterogeneity analysis and sensitivity analysis. According to the Cochrane handbook, Q-values $<$ degree of freedom (DF), $\mathrm{P}$-value $0.10, \mathrm{I}^{2}$-value between $0 \%$ to $40 \%$, considered might not be important heterogeneity, If Q-value $>$ DF, P-valuej0.10, I2-value between $75 \%$ to $100 \%$, considerable heterogeneity. ${ }^{14}$ Sensitivity analysis uses methods that compare fixed-model and random-model outcomes, if the results show no significant change in the fixed model, the sensitivity was low, and the results are relatively stable and reliable. Tests for funnel plot asymmetry should be used when there were at least 10 studies included in the NMA, ${ }^{14}$ Therefore, no publication bias analysis was conducted in this NMA.

\section{RESULTS}

\section{Characteristics of the included studies}

The search produced 745 citations, and 21 eligible articles were retrieved in full text. Six cluster RCTs were included after screening (Figure 1). ${ }^{6,10,15-18}$ In total, 11,828 HCWs were analyzed with a network meta-analysis, including 3 two-arm studies, and 3 three-arm studies (Table 2). In the included literature, we 
studied five kinds of interventions and one control group. The average age of the HCWs was 38.52 +- 11.67 $\left(10,387 \mathrm{HCWs}\right.$, five articles included, one article not reported $\left.{ }^{16}\right)$, and the sex ratio was $0.18(10,387$ HCWs, male 1,566 , female 8,821 , five articles included, one article not reported $\left.{ }^{16}\right)$.

\section{Risk-of-Bias and Quality-of-Evidence Assessment}

The research included in this study was used RevMan software (v 5.3) for the risk of bias summary. All of them were designed as cluster randomized controlled studies. All studies used random allocation concealment. Two studies reported as double-blinded (Figure 2) ${ }^{6,18}$.

\section{The incidence network meta-analysis by a laboratory-confirmed viral respiratory infection}

A total of six cluster RCTs were included in this NMA. There were 3 two-arm studies and 3 three-arm studies. A total of 6 nodes were included in this NMA, with each node represents a different rPPE and a wearing manner. The size of each node represents the included number of HCW for the intervention. The width of each line represents the number of direct comparisons between interventions (Figure 3A). The most studied interventions were continuous wearing surgical masks (4 RCTs) and continuous wearing N95 Respirator (3 RCTs).

In the NMA, Node splitting analysis shows P-value is 0.96 (Table 3), so we used a random model and the consistency type to analyze data. After 100,000 simulation iterations, the PSRF (potential scale reduction factor) value is 1 , indicating that approximate convergence has been reached. Pooled network OR values indicate that continuous wearing of N95 respirator (network OR, $0.48 ; 95 \%$ CrI 0.27 to 0.86 ) showed significantly superior over the control group (Figure 3C). Forest plot of the network meta-analysis comparing the efficacy of each rPPE class against the control group (Figure 3B). SUCRA analysis provided a ranking of each $\mathrm{rPPE}$ and a wearing manner according to its efficacy in reducing the incidence of laboratory-confirmed viral respiratory infection (Figure 3C). the top-ranked rPPE was the continuous wearing of N95 respirator (SUCRA score, 85.4).

Heterogeneity analysis shows no significant heterogeneity $((\mathrm{Q}$-value $=1.32<4(\mathrm{DF}), \quad \mathrm{P}$-value $=0.86$, I2value $=0 \%)$. The sensitivity analysis was conducted, and results showed that there is no significant change in fixed models (Supplementary Figure 1A\&B). Hence, the sensitivity was low, and the results were stable.

\section{The incidence network meta-analysis by clinical respiratory illness}

A total of five cluster RCTs were included in this NMA. There were 3 two-arm studies and 3 three-arm studies. A total of 6 nodes were included in the efficiency of the incidence of clinical respiratory illness network metaanalysis, with each node represents a different $\mathrm{rPPE}$ and wearing manner. The size of each node represents the included HCWs for the intervention. The width of each line represents the number of direct comparisons between interventions (Figure 4A). The most studied interventions were continuous wearing surgical masks (3 RCTs) and targeted wearing N95 Respirator (3 RCTs). In the NMA, Since the Node splitting analysis can't run, we provide both consistency type and inconsistency type data (Supplementary Figure 2 A\&B) Pooled network OR values indicate that no intervention was significantly superior over the control group (Figure 3C). Forest plot of the network meta-analysis comparing the CRI of each rPPE class against the control group (Figure 4B). SUCRA analysis provided a ranking of each rPPE and wearing manner according to its incidence of CRI (Figure 4C). Although all classes were equivalent to control, the top-ranked rPPE was the continuous wearing of N95 respirator (SUCRA score, 79.5).

Heterogeneity analysis shows no significant heterogeneity ((Q-value $=0.73<2(\mathrm{DF}), \mathrm{P}$-value $=0.69$, I2value $=0 \%)$ ), The sensitivity analysis was conducted and the results showed that the $95 \% \mathrm{CrI}$ has a significant change in fixed and random models (Supplementary Figure 2C\&D). Hence, the sensitivity was high, and the results were unstable. 


\section{Discussion}

COVID-19 is predominantly transmitted by contact or droplet. Airborne transmission may occur if the patient had respiratory symptoms such as coughing or HCWs performing high-risk procedures liked incubation. ${ }^{19}$ Preventing VRID transmission by rPPE is highly recommended. But current guidelines for the use of rPPE in HCWs in the hospital setting are based on limited evidence-based studies ${ }^{20}$ In this NMA of 6 RCTs consisting of 11,828 HCWs, and we compared the protective effect of three types of rPPE. Additionally, we focused on the wearing manner for further assessment. Results of NMA showed that continuous wearing of N95 respirator on the whole shift may have better protection against VRID, whereas there is no significant difference in the CRI.

Appropriate rPPE use is critical to decreasing the infectious risk for HCWs. However, previous RCTs showed inconsistent results in different rPPE. ${ }^{21,22}$ Our finding supports that the N95 respirator is superior to surgical mask and cloth mask. Furthermore, continuous wearing showed an increasingly protective effect against VRID. In the medical setting such as in the emergency medicine department, patients with VRID are not able to be screened or confirmed by serological tests or medical imagining. HCWs who exposed to such an environment will face a higher risk of VRID. In the COVID-19 epidemic, asymptomatic carriers had been proved to be contagious ${ }^{8}$, which also became a potential risk for HCWs. Hence, the continuous wearing of the N95 respirator during the whole shift might provide more consistent and reliable protection for HCWs.

There is an unexpected result is that the targeted wearing of surgical masks showed better efficacy than the continuous wearing of it. One explanation could be the moist condensation from exhalation to the inner surface of musk would decrease the filtration rate with prolonged and continuously wearing time. ${ }^{23}$ Reusable cloth mask, which is widely used in the underserved area, showed only marginal protection against VRID. Lack of proper guidelines and equipment to decontaminate reusable cloth mask could contribute to this because the airborne pathogen can survive on the mask surface for days. Besides, the cloth mask showed lower filtration capacity than disposable masks. ${ }^{24}$

The sensitivity analysis of the incidence of clinical respiratory illness is unstable (Supplementary figure 2 ). So, we assumed there is no rPPE is superior to the control group in preventing VRID (Figure 3C). We speculated that factors that drive other bias are difficult to quantify. Therefore, our finding of the aforementioned rPPEs in preventing VRID by the incidence of CRI should be cautiously interpreted.

There are some other limitations to our analysis. First, the consistency evaluation for the protective efficacy of rPPE may vary in different studies. Different medical settings might have different risks of infection. For example, HCWs in emergency settings are more susceptible to VRID due to the crowded environment comparing to a well-controlled surgical/operation setting. To better evaluate the protection level of rPPE, it is preferring to evaluate PPEs under the same working environment and treating similar patient groups. Second, there is a limited quantity of studies that focus on rPPE has published during this COVID-19 epidemic. So, the reliability of selection in this study is relatively lacking. And the incidence of CRI network meta-analysis results in Sensitivity analyses shows different confidence intervals, this indicates other bias, but we failed to find the source of bias. therefore, the results should be dealt with some caution. Also, any biases and inaccuracies within individual studies would undermine our analysis. Further analysis should include more RCTs during this COVID-19 epidemic.

\section{Conclusion}

This NMA showed that continuous wearing of N95 respirator on the whole shift may have the best protection against VRID. Surgical Mask, on the contrary, needs to be replaced frequently for better efficacy. In terms of cloth mask, although it is still in being used, only provides marginal protection against VRID. Further analysis should include more RCTs during this COVID-19 epidemic. 


\section{References}

1. Bein B, Bachmann M, Huggett S, Wegermann P. [SARS CoV-2/COVID-19: Evidence-Based Recommendation on Diagnosis and Therapy].Anasthesiol Intensivmed Notfallmed Schmerzther.2020;55(4):257-265.

2. Allyson, Lipp. Disposable surgical face masks for preventing surgical wound infection in clean surgery. Journal of Evidence-Based Medicine. 2012.

3. Janssen L, Ettinger H, Graham S, Shaffer R, Zhuang Z. The Use of Respirators to Reduce Inhalation of Airborne Biological Agents.Journal of Occupational \& Environmental Hygiene.2013;10(8):D97-D103.

4. Vittoria O, Fu YC, Fong LMS, Tam CC. Effectiveness of Masks and Respirators Against Respiratory Infections in Healthcare Workers: A Systematic Review and Meta-Analysis. Clinical Infectious Diseases. 2017(11):11.

5. Bartoszko JJ, Farooqi MAM, Alhazzani W, Loeb M. Medical Masks vs N95 Respirators for Preventing COVID-19 in Health Care Workers A Systematic Review and Meta-Analysis of Randomized Trials. Influenza and Other Respiratory Viruses.

6. Radonovich LJ, Jr., Simberkoff MS, Bessesen MT, et al. N95 Respirators vs Medical Masks for Preventing Influenza Among Health Care Personnel: A Randomized Clinical Trial. JAMA.2019;322(9):824-833.

7. Vanhems P, Benet T, Munier-Marion E. Nosocomial influenza: encouraging insights and future challenges. Current Opinion in Infectious Diseases. 2016;29(4):366.

8. Hu Z, Song C, Xu C, Jin G, Shen H. Clinical Characteristics of 24 Asymptomatic Infections with COVID19 Screened Among Close Contacts in Nanjing, China. SSRN Electronic Journal. 2020.

9. Thiberville SD, Salez N, Benkouiten S, Badiaga S, Charrel R, Brouqui P. Respiratory viruses within homeless shelters in Marseille, France.Bmc Research Notes. 2014;7.

10. MacIntyre CR, Wang Q, Seale H, et al. A randomized clinical trial of three options for N95 respirators and medical masks in health workers. Am J Respir Crit Care Med. 2013;187(9):960-966.

11. Hutton B, Salanti G, Caldwell DM, et al. The PRISMA extension statement for reporting of systematic reviews incorporating network meta-analyses of health care interventions: checklist and explanations. Ann Intern Med. 2015;162(11):777-784.

12. Dias S, Welton NJ, Caldwell DM, Ades AE. Checking consistency in mixed treatment comparison meta-analysis. Statistics in Medicine.2010;29(7-8):932-944.

13. Brooks SP, Gelman A. General Methods for Monitoring Convergence of Iterative Simulations. Journal of Computational and Graphical Statistics. 1998;7(4):434-455.

14. Higgins JPT, Thomas J, Chandler J, Cumpston M, Welch VA. Cochrane Handbook for Systematic Reviews of Interventions. 2019.

15. MacIntyre CR, Seale H, Dung TC, et al. A cluster randomised trial of cloth masks compared with medical masks in healthcare workers. BMJ Open. 2015;5(4):e006577.

16. MacIntyre CR, Wang Q, Rahman B, et al. Efficacy of face masks and respirators in preventing upper respiratory tract bacterial colonization and co-infection in hospital healthcare workers. Prev Med.2014;62:1-7.

17. MacIntyre CR, Wang Q, Cauchemez S, et al. A cluster randomized clinical trial comparing fit-tested and non-fit-tested N95 respirators to medical masks to prevent respiratory virus infection in health care workers. Influenza Other Respir Viruses. 2011;5(3):170-179.

18. Loeb M, Dafoe N, Mahony J, et al. Surgical mask vs N95 respirator for preventing influenza among health care workers: a randomized trial.JAMA. 2009;302(17):1865-1871. 
19. Tian S, Hu N, Lou J, et al. Characteristics of COVID-19 infection in Beijing. J Infect. 2020;80(4):401406.

20. Nicola M, O'Neill N, Sohrabi C, Khan M, Agha M, Agha R. Evidence based management guideline for the COVID-19 pandemic - Review article.Int J Surg. 2020;77:206-216.

21. Atrie D, Worster A. Surgical mask versus N95 respirator for preventing influenza among health care workers: a randomized trial. CJEM. 2012;14(1):50-52.

22. Chen X, Chughtai AA, MacIntyre CR. Herd protection effect of N95 respirators in healthcare workers. $J$ Int Med Res.2017;45(6):1760-1767.

23. Barbosa MH, Graziano KU. Influence of wearing time on efficacy of disposable surgical masks as microbial barrier. Brazilian Journal of Microbiology. 2006;37(3):216-217.

24. Quesnel LB. The efficiency of surgical masks of varying design and composition. British Journal of Surgery. 1975;62(12):936-940.

Table 1. Characteristics of the laboratory-confirmed viral respiratory infection and clinical respiratory illness

\section{Study ID Laboratory-confirmed viral respiratory infection}

Loeb 2008-09

MacIntyre 2008-09

MacIntyre 2008-09 IORV

MacIntyre 2009-10

MacIntyre 2011

Radonovich 2011-15 parainfluenza, influenza viruses A and B, Respiratory syncytial virus, Metapneumovirus, Rhino Adenoviruses, human metapneumovirus, coronaviruses, parainfluenza viruses, influenza viruses Adenoviruses, human metapneumovirus, coronavirus, parainfluenza viruses 1, 2 and 3 , influenza Adenoviruses, human metapneumovirus, coronaviruses parainfluenza viruses, influenza viruses $A$ respiratory syncytial virus (RSV) A and B, human metapneumovirus (hMPV), influenza A and Coxsackie/echoviruses, Coronavirus, Human metapneumovirus, Human rhinovirus, Influenza A

IORV:without control arm

Table 2 Characteristics of the included studies

\begin{tabular}{llllll}
\hline Study ID & Country & Study size & Sex(Male/Female) & Age(years) & (allocated numbers in arms) \\
\hline Loeb 2008-09 & Canada & 446 & $26 / 420$ & $36.15 \pm 10.59$ & Targeted wearing surgical mask (225) vs \\
MacIntyre 2008-09 & China & 1441 & N/A & N/A & Continuous wearing N95 Respirators ma \\
MacIntyre 2008-09 & China & 1485 & $142 / 1343$ & $32.85 \pm 9.32$ & Continuous wearing surgical mask (492) \\
MacIntyre 2009-10 & China & 1669 & $243 / 1426$ & $33.1 \pm 9.61$ & Continuous wearing surgical mask(572) \\
MacIntyre 2011 & Vietnam & 1607 & $357 / 1250$ & $35.65 \pm 10.39$ & Continuous wearing sugical masks (580) \\
Radonovich 2011-15 & America & 5180 & $798 / 4382$ & $43 \pm 11.55$ & Targeted wearing N95 Respirator(2512)v \\
\hline
\end{tabular}

Table 3. Node-splitting analysis for incidence of laboratory-confirmed viral respiratory infection

\begin{tabular}{lllll}
\hline Name & Direct Effect & Indirect Effect & Overall & P-Value \\
\hline continuous wearing of N95 respirator, control & $0.76(0.01,1.60)$ & $0.72(-0.26,1.77)$ & $0.74(0.16,1.31)$ & 0.96 \\
\hline
\end{tabular}

Figure Legends

Figure 2. Risk of bias summary. IORV:without control arm

Figure 3. The incidence network meta-analysis by a laboratory-confirmed viral respiratory infection. (A) The network plot shows a comparison of the incidence of the laboratory-confirmed viral respiratory infection between nodes (blue circles). Each node represents a unique rPPE and wearing manner 
or control, the size of each node represents the included HCWs for the intervention. The width of each line represents the number of direct comparisons between interventions. The connecting line was noted the number of trial-level comparisons between the two nodes. (B) The forest plot of the network meta-analysis comparing the VRID of each rPPE group against the control group. (C) Schematic detailing the most efficacious $\mathrm{rPPE}$ class in terms of reducing laboratory-confirmed viral respiratory infection according to the surface under the cumulative ranking curve analysis (SUCRA). OR, Odd ratio. CrI, credibility interval. rPPE, respiratory personal protective equipment.

Figure 4. The incidence network meta-analysis by clinical respiratory illness. (A) Network plot showing comparisons of the incidence of clinical respiratory illness between nodes (blue circles), each representing a unique rPPE and wearing manner or control, the size of each node represents the included HCWs for the intervention. The width of each line represents the number of direct comparisons between interventions, the connecting line was noted the number of trial-level comparisons between the two nodes. (B)Forest plot of the network meta-analysis comparing the CRI of each rPPE group against the control group. (C) Schematic detailing the most efficacious rPPE classes in terms of reducing CRI according to the surface under the cumulative ranking curve analysis (SUCRA). OR, Odd ratio. CrI, credibility interval. rPPE, respiratory personal protective equipment.

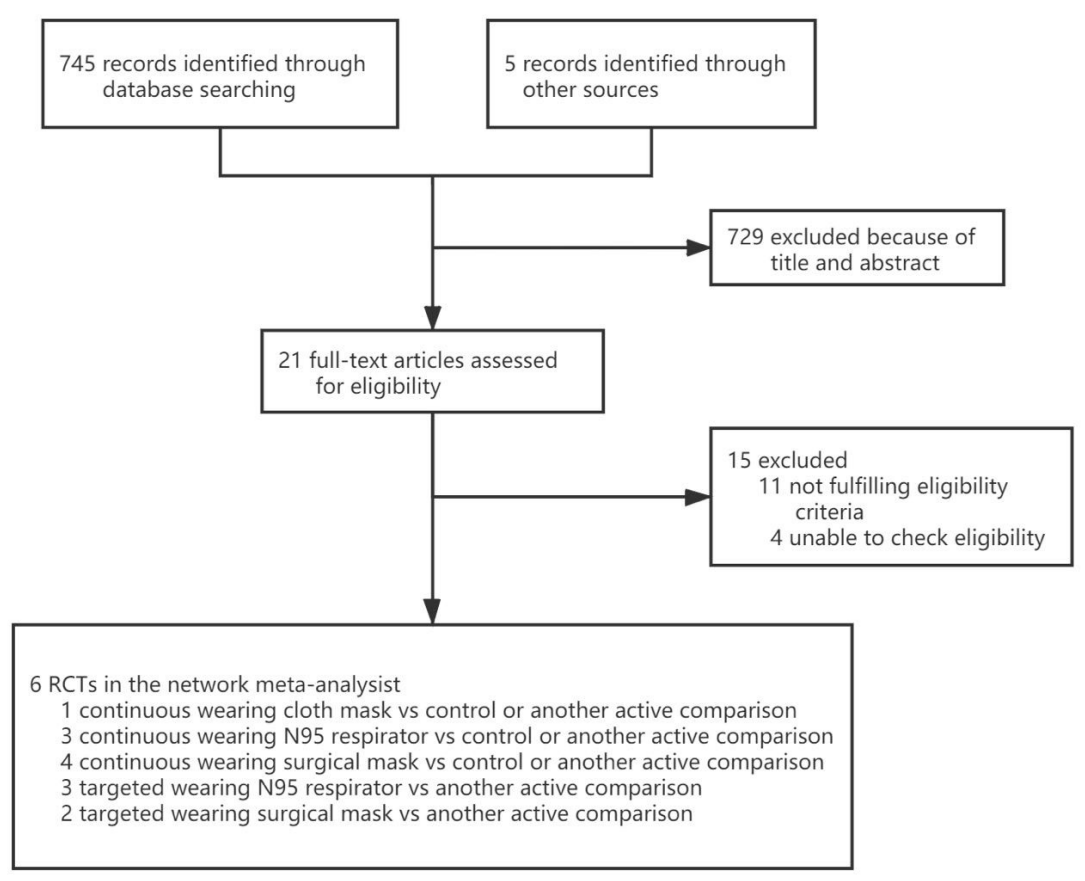




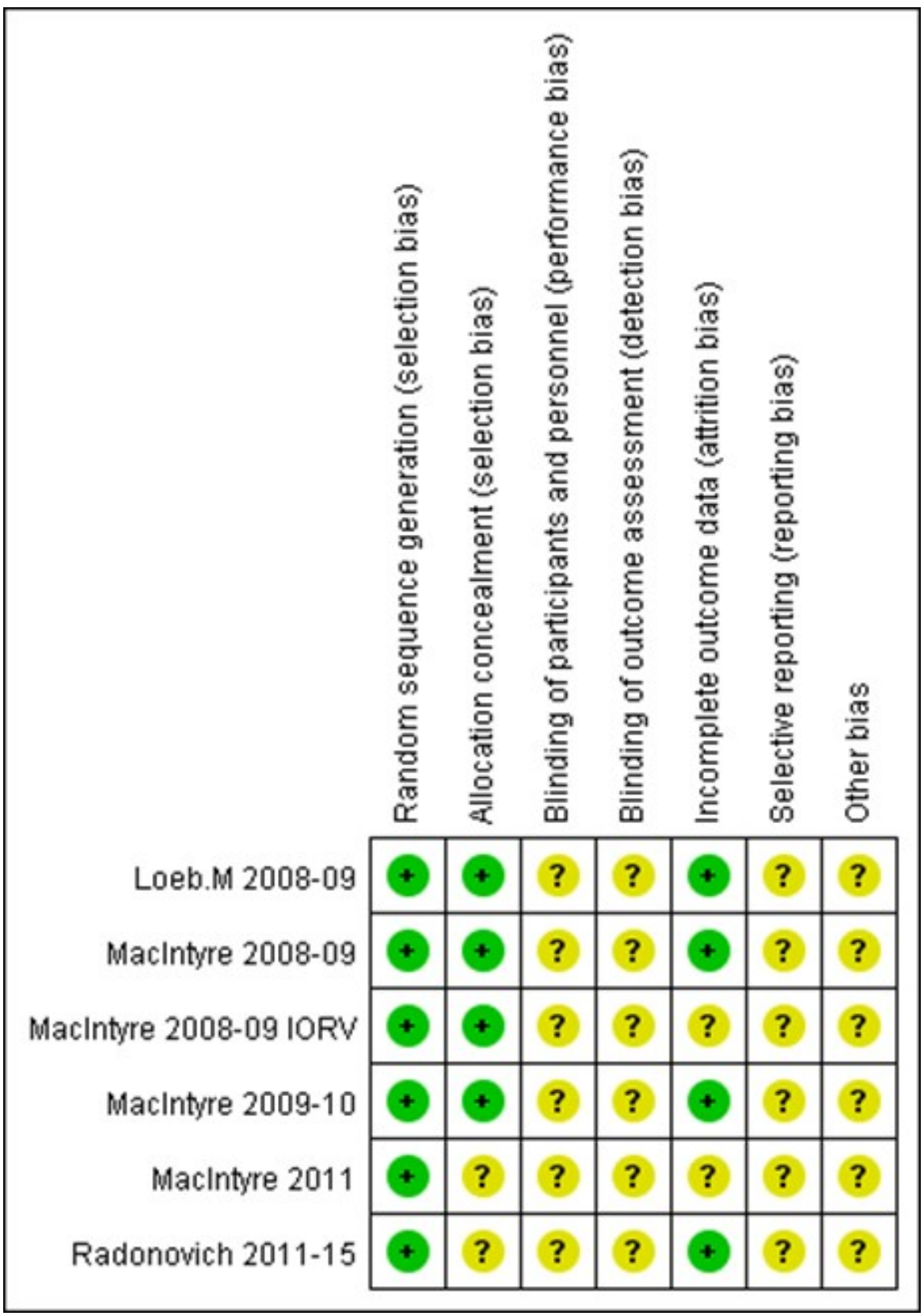



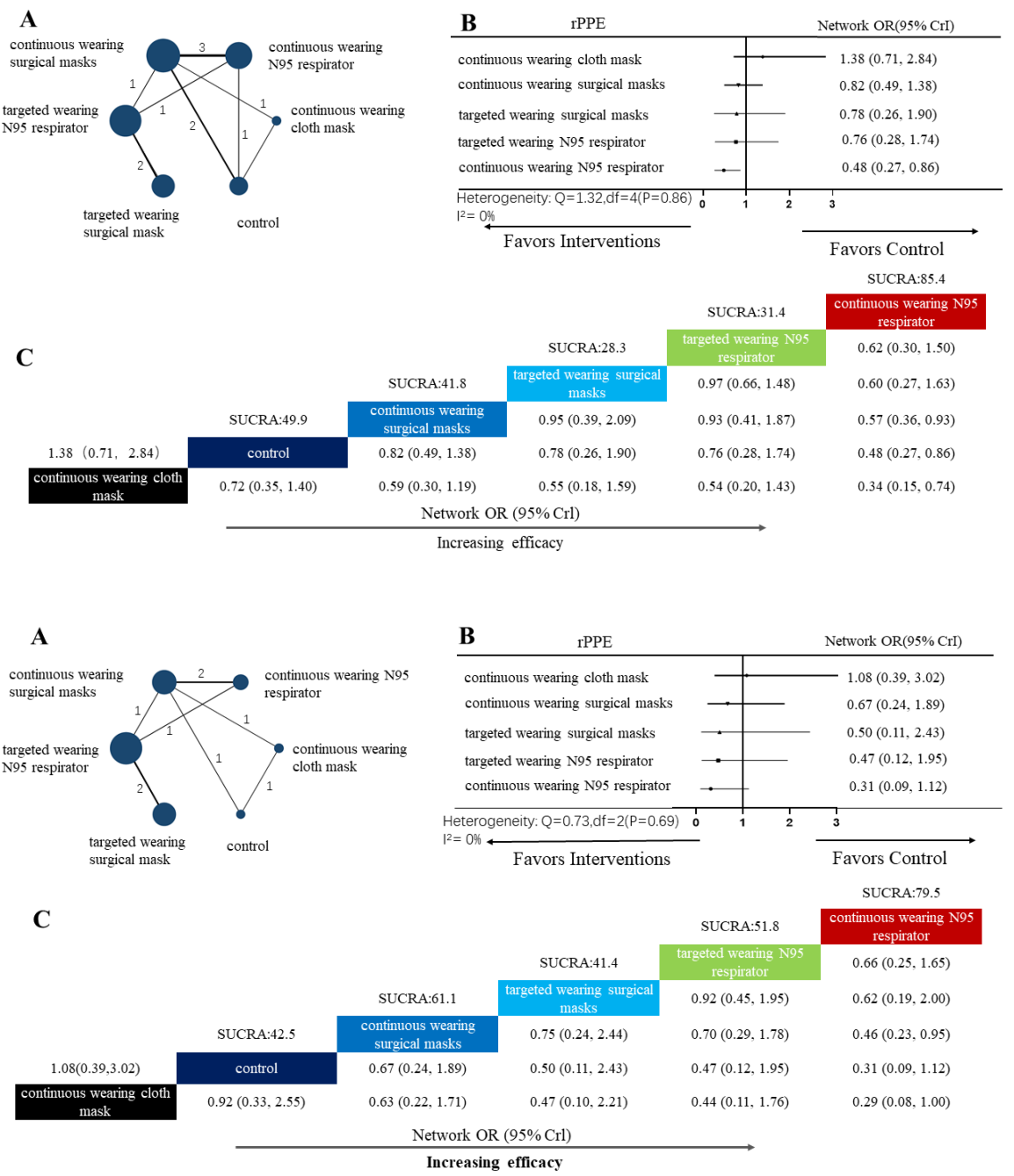

\section{Hosted file}

Table 1. Characteristics of the laboratory-confirmed viral respiratory infection and clinical respirato available at https://authorea.com/users/347779/articles/473325-comparative-efficacy-ofrespiratory-personal-protective-equipment-against-viral-respiratory-infectious-diseasesin-healthcare-workers-a-network-meta-analysis

\section{Hosted file}

Table 2. Characteristics of the included studies.docx available at https://authorea.com/ users/347779/articles/473325-comparative-efficacy-of-respiratory-personal-protectiveequipment-against-viral-respiratory-infectious-diseases-in-healthcare-workers-a-networkmeta-analysis

\section{Hosted file}

Table 3. Node-splitting analysis for incidence of laboratory-confirmed viral respiratory infection.docx available at https://authorea.com/users/347779/articles/473325-comparative-efficacy-ofrespiratory-personal-protective-equipment-against-viral-respiratory-infectious-diseasesin-healthcare-workers-a-network-meta-analysis 